\title{
A New Haptic Sensor Actuator System for Virtual Reality Applications in Medicine
}

\author{
Walaa Khaled ${ }^{1}$, Stefan Reichling ${ }^{1}$, Otto T. Bruhns ${ }^{1}$, Holger Boese ${ }^{2}$, \\ Mario Baumann ${ }^{2}$, Gareth Monkman ${ }^{3}$, Stefan Egersdoerfer ${ }^{3}$, \\ Herbert Freimuth ${ }^{4}$, and Helmut Ermert ${ }^{1}$ \\ ${ }^{1}$ Ruhr-University Bochum, Universitaetsstr. 150, IC-6/133, \\ 44780 Bochum, Germany \\ Walaa.Khaledarub. de \\ ${ }^{2}$ Fraunhofer-Institut fuer Silicatforschung ISC, Neunerplatz 2, \\ 97082 Wuerzburg, Germany \\ ${ }^{3}$ Fachhochschule Regensburg, PruefeningerStr. 58, \\ 93049 Regensburg, Germany \\ ${ }^{4}$ Institute fuer Mikrotechnik Mainz GmbH, Carl-Zeiss-Str. 18-20, \\ 55129 Mainz, Germany
}

\begin{abstract}
The pathological state of soft tissues is often correlated with changes in stiffness. Malignant tumors are significantly stiffer and more immobile than surrounding healthy tissue. (hard lesions, "nodes" in organs: tumors; calcifications in vessels: arteriosclerosis). The main problem is, that such information is usually not available or can only be obtained by manual palpation, which is subjective and limited in sensitivity. It requires intuitive assessment and does not allow quantitative documentation. On the one hand a suitable sensor is required for quantitative measurement of mechanical tissue properties. On the other hand, there is also a need for a realistic mechanical display of such tissue properties. Suitable actuator arrays with high spatial resolution acting in real time are required. A haptic sensor actuator system is presented in this paper including a sensitive sensor part and an actuator array for different applications. The mechanical consistency of an object is to be locally specified using a sensor system and represented perceptibly in a remote position on a tactile display (actuator system) for the user. The sensor system uses ultrasound (US) elastography, whereas the actuator array is based on electrorheological (ER) fluids.
\end{abstract}

\section{Introduction}

Real time US elastography represents a recent development to determine strain and elasticity distributions inside objects scanned [1]. Commonly used imaging techniques rely on the interpretation of two dimensional visual data displayed on a video screen. In addition to visual data, a physician will employ tactile exploration making the simultaneous portrayal of both video and haptic information most desirable [2]. In the last few years, interest has been directed toward the display of pictures on haptic surfaces [3-4]. Such an imaging system would allow surgeons to document properties of hard lumps contained in soft tissue, and to assist in operations performed remotely. 
The general design of the haptic system is presented in the next section of this paper. Some fundamental principles and methods of the real time ultrasound elastography and the tactile actuator design are described in further sections.

\section{Haptic System Description}

Four German institutes are operating in a collaborative project, developing a haptic system that consists of a sensor head and a separate actuator array, as shown in Fig. 1. The sensor system is based on real time ultrasound elastography and is able to detect even small and far surface lesions which are not detectable by manual palpation or conventional US systems. The actuator consists of a tactile display, which has a hybrid configuration consisting of smart fluids with electrically controlled rheological properties inside micro-machined cells. The ultrasonic device generates and transfers images to the high voltage control unit. The control unit uses a newly designed optocoupler to separate the touchable surface from the high voltage unit. The touchable surface of the actuator array consists of numerous small ER fluid cells. The elements of this actuator array are controlled by voltages which can be individually, and remotely, adjusted. By this means variable stiffness of the tactile elements is generated and locally varying consistency of the surface is perceived by the user who presses his fingers onto the virtual object. Due to their small size and their repetitive arrangement, the actuator elements, including the electrodes for the ER fluid, have to be manufactured using micro-mechanical production methods.

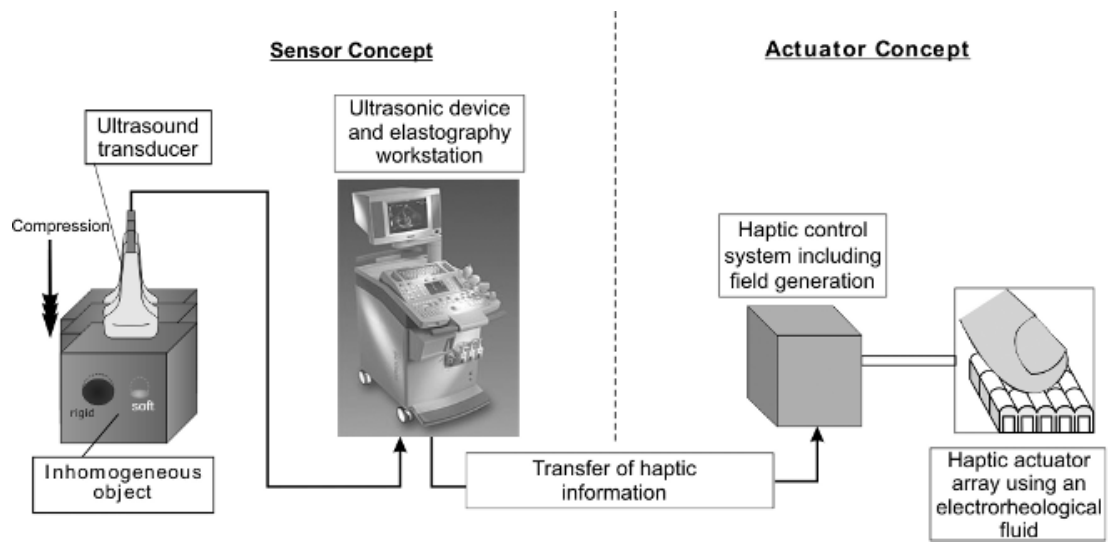

Fig. 1. Scheme of the haptic sensor-actor system

The required field strength for influencing the consistency of the ER fluid is about $2 \mathrm{kV} / \mathrm{mm}$ and needs a sophisticated system of high voltage distribution for individual actuator cell control. In order to achieve the required changes in stiffness, the evaluation of ER fluids and actuator designs together with various modes of operation are being investigated. The spatial haptic resolution of the final actuator array is few millimeters, which corresponds to the resolution of receptors on the finger tip. The com- 
bined sensor-actuator system will serve as a new technology from which the potential for various applications may be deduced.

\section{Sensor Based on Real Time Elastography}

Conventional diagnostic imaging modalities (X-ray, US, MRI) are not able to visualize the mechanical tissue properties directly. The standard screening procedure for the detection of breast, thyroid, prostate, and liver abnormalities is palpation. The pathological state of soft tissues is often correlated with changes in stiffness, which yields qualitative estimation of tissue elasticity characteristics. However, palpation is not very accurate because of its poor sensitivity with respect to small and deeply located lesions as well as to its limited accuracy in terms of the morphological localization of lesions. Therefore, elastography as a new method based on US or on magnetic resonance imaging techniques (MRI) is of growing interest because of the capability of elastic tissue property visualization. In addition to tumors in soft tissue elastography is also able to detect calcifications in blood vessels, for example in the coronary arteries. The advantages of US elastography over MRI elastography are easier applicability and real time capability. Real time US elastography has been recently developed based on high efficiency signal processing approaches [5].

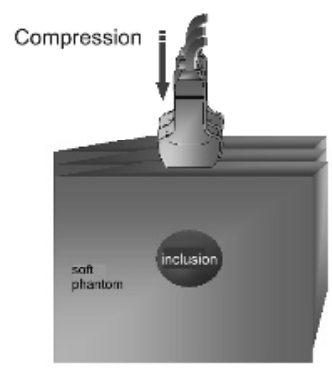

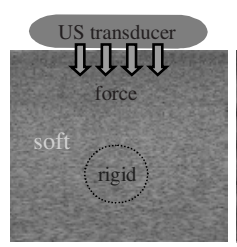

B-mode image

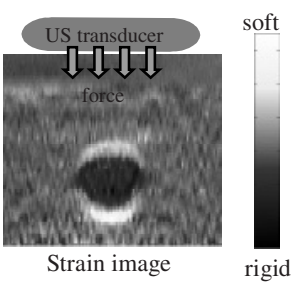

Strain image

Fig. 2. A tissue mimicking soft phantom with a hard inclusion is slightly compressed using the ultrasound transducer (far left). Because of uniform reflection of the isoechoic inclusion, the rigid body with circular shape in the phantom is not visible in the conventional B-Mode image. The same body can be visible in the elastogram (strain image) to the right.

Ultrasonic imaging is performed during compression of the medium by an external force, as shown in Fig. 2, to determine the strain distribution. A strain image is formed by comparing echo signal sets obtained prior to and immediately following less than $1 \%$ compression of the height, where tissue mechanical properties are approximately linear and elastic. Using the exact measurement of temporal displacements between the two signal sets is the key to estimate strain. A phase root seeking algorithm has been developed [5] for a fast and accurate displacement shift estimation, improving the accuracy, reducing the time needed and establishing the first freehand, real time, two dimensional US elastography system. During a clinical study [6], radio-frequency ultrasonic echo data using B-Mode and strain image mode of more than 216 patients is undergoing clinical examinations. It has been shown, that our 
system for real time ultrasound elastography is able to detect the prostate carcinoma with a high grade of accuracy reaching a sensitivity of $76 \%$ and a specificity of $84 \%$, compared with only $34 \%$ using ultrasound B-Mode images alone. Thereby the system can improve the early detection of prostate cancer and allow a more reliable diagnosis. Figure 3 shows an example, where prostate slices with histological diagnosis following radical prostatectomies act as the gold standard. Cancerous areas have been stained and marked on the prostate slices.

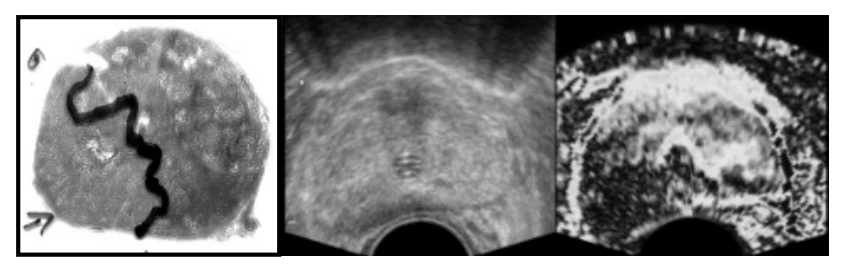

(a)

(b)

(c)

Fig. 3. In vivo results of a human prostate: (a) Histology, tumors have been stained, malign and benign tissue areas have been marked by pathologist. The tumor is in the lower left side marked with an arrow (b) It is hardly visible in the B-Mode image. (c) The tumor is clearly visible as a dark left area on the strain image.

In an experiment, as shown Fig. 4, an ultrasonic tissue mimicking sponge Phantom including a harder embedded agar inclusion, whose largest axis was $7 \mathrm{~mm}$, was imaged using the elastography system. The ultrasound RF-data were acquired at $1 \mathrm{~mm}$ intervals, using a commercially available ultrasound system modified to get RF-signal and sampled by a conventional ADC-card and a desktop PC. The axial strains were calculated using the fast phase root-seeking technique to establish strain images. In order to develop inverse elastography reconstruction approaches, finite element simulations were performed for a number of soft biological tissue models. The results obtained from finite element analysis were confirmed in the ultrasonic experiments on a set of tissue-mimicking phantoms with known acoustical and mechanical properties. Finally, using numerical solution models and solving the inverse problem we can deduce relative mechanical properties. For the reprojection of $2 \mathrm{D}$-freehand-slices, as shown in Fig. 4, into a volume data set a position sensing device is used. The position data acquired with each 2D elastogram determines the particular location of the image. After acquiring a series of $2 \mathrm{D}$ elastograms, the volume is created by placing each image at the proper location in the volume.

Some physicians assert that the correct estimation of the tumor volume (in vivo) for example in prostate cancer would be helpful in formulating treatment for the disease, since (ex vivo) determination of tumor volume has been shown to correlate with the progression of the disease after radical prostatectomy [7]. Using the three dimensional volume data in reconstructing an equivalent virtual object displayed on the actively controllable 3D object surface is the aim of the tactile display. 

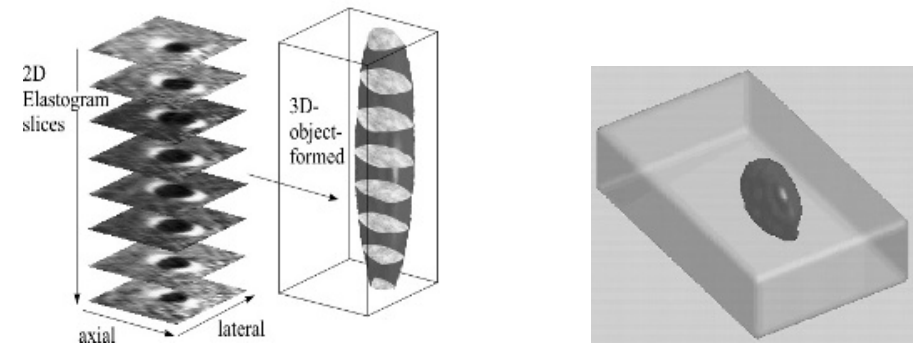

Fig. 4. Sequential acquisition of parallel tomographic slices using Elastography, combined with image segmentation, enables the reconstruction of $3 \mathrm{D}$ image to the right.
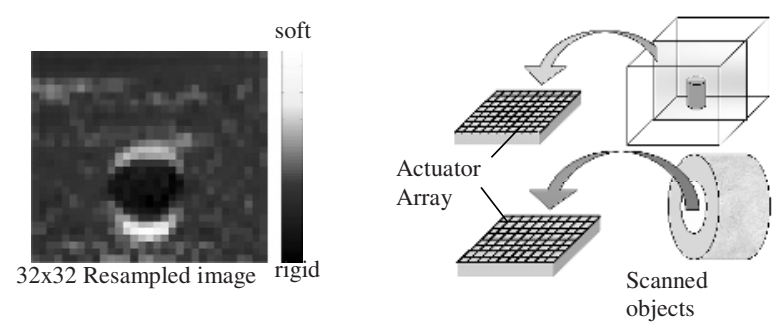

Fig. 5. Using resampling techniques the visual image can be reproduced on a haptic actuator array with $32 \times 32$ elements.

Any $2 \mathrm{D}$ projections can be selected from the $3 \mathrm{D}$ data set for the presentation on a tactile display system. Using a tactile display with an appropriate resolution can improve diagnostic and therapeutic methods. An actuator array is envisioned to consist of 1024 (32x32) elements, as shown in Fig. 5, presenting elastographic images, resampled by spatially filtering, which can allow physicians to palpate areas not detectable using common methods and easily determine the volume of Tumors.

\section{Tactile Actuator with ER Fluid}

Numerous forms of tactile interfaces for virtual environments have been researched to date, including investigations in how to provide contact force, slip, texture, vibration, and thermal sensation. Any form of tactile display must deliver a good spatial resolution, because of the tactile sensitivity of the fingers. This can be achieved using a matrix of elements, which provide a stimulus in the form of physical movement or vibration in the vertical plane proportional to the elasticity of the object being portrayed. 
Table 1. Actuators previously used in tactile displays and the ER fluid array

\begin{tabular}{|l|l|l|l|l|l|l|}
\hline Physical actuation & Array size & Force & Hor. Res. & Ver. stroke & Rep. freq. Power/unit \\
\hline Pneumatic air jet & $7 \times 7$ & - & $6.5 \mathrm{~mm}$ & - & $2 \mathrm{~Hz}$ & $1 \mathrm{~W}$ \\
\hline Pneumatic cylinder & $4 \times 4$ & $2 \mathrm{~N}$ & $4 \mathrm{~mm}$ & $10 \mathrm{~mm}$ & $10 \mathrm{~Hz}$ & $0.3 \mathrm{~W}$ \\
\hline Electromag. vibrator & $20 \times 20$ & $0.5 \mathrm{~N}$ & $12 \mathrm{~mm}$ & $10 \mathrm{~mm}$ & $100 \mathrm{~Hz}$ & $1 \mathrm{~W}$ \\
\hline Piezoelectric vibrator & $24 \times 6$ & $0.4 \mathrm{~N}$ & $1.5 \mathrm{~mm}$ & $5 \mathrm{~mm}$ & $300 \mathrm{~Hz}$ & $10^{-5} \mathrm{~W}$ \\
\hline Thermal shape & $8 \times 8$ & $2.5 \mathrm{~N}$ & $2 \mathrm{~mm}$ & $2.5 \mathrm{~mm}$ & $<0.3 \mathrm{~Hz}$ & $0.5 \mathrm{~W}$ \\
\hline Pulsed Elec. simulation & $20 \times 20$ & - & $5 \mathrm{~mm}$ & - & $400 \mathrm{~Hz}$ & $0.01 \mathrm{~W}$ \\
\hline ER fluid array & $24 \times 24$ & $1 \mathrm{~N}$ & $2.4 \mathrm{~mm}$ & $2 \mathrm{~mm}$ & $>50 \mathrm{~Hz}$ & $0.02 \mathrm{~W}$ \\
\hline
\end{tabular}

Table 1 shows actuation methods which have hitherto been used and the ER fluid array. In order to limit the physical size, have a good resolution and an optimal power consumption of the actuator, an ER fluid array is being used. ER fluids behave as normal Newtonian liquids until subjected to a strong electric field, then they undergo a phase change, as shown in Fig. 6, from liquid to a quasi-solid state. Electrorheological fluids are suspensions of electrically polarizing particles in a nonconducting carrier liquid. The effective consistency of the ER fluid increases with the application of a strong electrical field. This effect is fast, reversible and can be easily exploited in the design and construction of tactile elements. Some of the advantages of ER fluids are their high shear stress, low current density and fast response. Convenient ER fluids are also nontoxic and non-polluting, meeting health and safety regulations. The flow of ER fluids can be controlled by simply passing the fluid between two electrodes (flow mode).
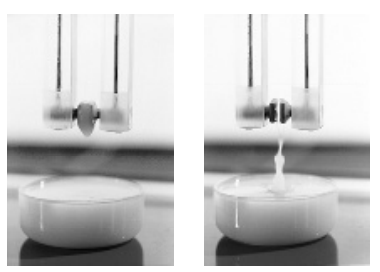

Fig. 6. An ER fluid in the presence of electric field changes consistency and internal friction. The ER fluid sticks between the charged electrodes (left), and drops down after switching the electrical field off (right).

This makes the design of hydraulic valves extremely simple and their construction very small. Another possibility is to switch micro-machined elements with the ER fluid integrated into the tactile display directly (shear mode). This has been done previously for binary (on/off) displays (squeeze mode) [8], and more recently with a degree of vertical resolution as shown in [9]. The goal of the present development is to generate an experimental model for the haptic sensor actuator system. In the first step, 
individual single components of the sensor-actuator-system were produced and tested. Test results from two shear mode versions, one with cylindrical elements and the other with planar elements, are shown in Table 2.

Table 2. New electrorheological actuators used in tactile displays

\begin{tabular}{|l|l|l|l|l|l|l|}
\hline Physical actuation & Array size & Force & Hor. Resol. & Ver. stroke & Bandwidth & Power/unit \\
\hline Cylindrical & $4 \times 4$ & $1.4 \mathrm{~N}$ & $5 \mathrm{~mm}$ & $30 \mathrm{~mm}$ & $>20 \mathrm{~Hz}$ & $20 \mathrm{~mW}$ \\
\hline Planar & $4 \times 4$ & $0.6 \mathrm{~N}$ & $3 \mathrm{~mm}$ & $40 \mathrm{~mm}$ & $>20 \mathrm{~Hz}$ & $27 \mathrm{~mW}$ \\
\hline
\end{tabular}

It can be seen that ER fluids enjoy a comparatively low power consumption. A total power consumption of less than 30 Watts for a 1024-element-array is required, which is much lower than for hydraulic or electromagnetic systems. Fig. 7 shows the scheme of an actuator element with the ER fluid in which a tubular piston is allowed to move. While pressing down the piston the ER fluid between the electrodes is subjected to shear forces while an upward flow is induced due to the displacement of the fluid below the piston. As a result, the motion of the piston generates a resistance force which depends on the consistency of the ER fluid which is in turn determined by the electrical field strength between the electrodes.

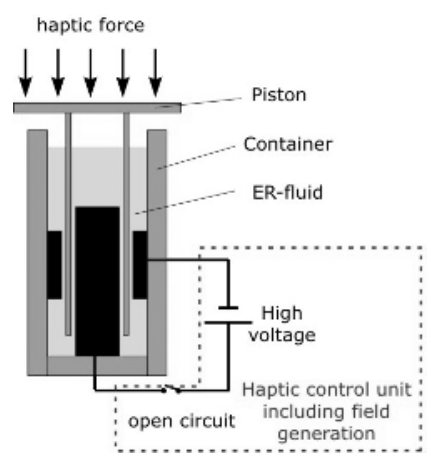

(a)

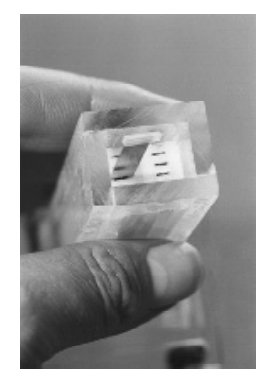

(b)

Fig. 7. a) Scheme of a haptic actuator element (side view) b) Actuator array unit (8 elements) with only one piston element (upper view)

The tactile element of Fig. 7 has been coupled with a force measurement device and a range of newly developed ER fluids have been investigated. An appropriate experiment was developed to measure the shear stress in the actuator, when using the piston, depending on the electrical Field as shown in Fig. 8 a). For the most convenient ER fluid a force of nearly $10 \mathrm{~N}$ could be achieved on the pistons of about $1 \mathrm{~cm}^{2}$ area at a field strength of $2 \mathrm{kV} / \mathrm{mm}$ as shown in Fig. $8 \mathrm{~b}$ ). 
(a)

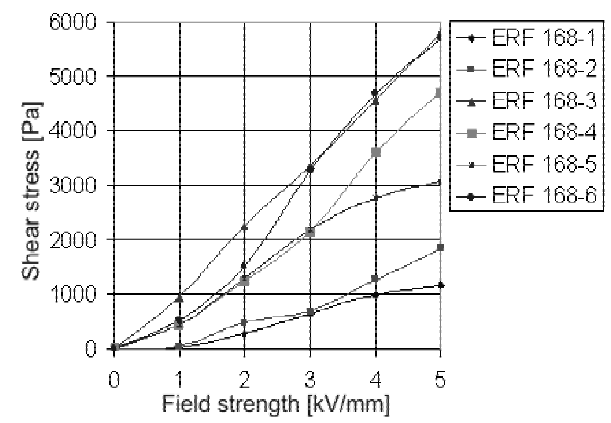

(b)

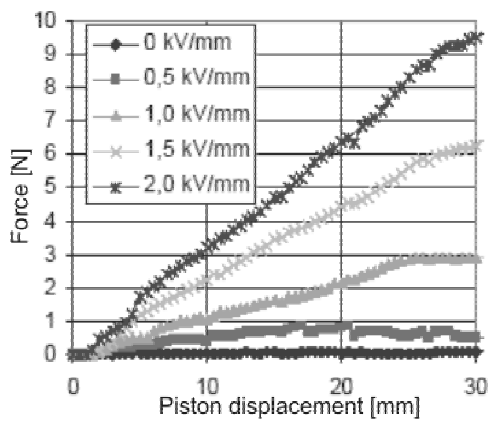

Fig. 8. Shear stress of various ER fluids measured for different electrical field strengths (DC voltage, shear rate $10 \mathrm{~s}^{-1}$, temperature $25^{\circ} \mathrm{C}$ )

A newly developed ER fluid, designated ERF 168-3, was selected as particularly suitable to use in the haptic actuator, due to its high shear stress at lower field strength requirements. The control of the actuators with voltages of up to $2 \mathrm{kV}$ and very small current flows required new switching concepts based on special light sensitive semiconductor materials [10]. Going from design to production several methods, processes and materials can be used to produce micro-structured products. For realization by hot embossing or injection molding it is necessary to produce mold inserts possessing the negative pattern of the final microstructure. Mold inserts can be produced for example by high precision milling for structure sizes down to $100 \mu \mathrm{m}$. By using a special plasma etching technique it is possible to fabricate silicon structures independent of the crystal orientation with depths of several hundred micrometers and high aspect ratios.

\section{Conclusion}

The fundamental model of a haptic sensor-actuator array system has been developed. This system allows on-line real time display of mechanical properties as well as off line display modes. This is the first integrated haptic sensor actuator system based on US elastography and ER fluids with interesting potentials of virtual reality applications in medicine. It can be used for medical teaching purposes, for applications in telemedicine, intraoperative applications like minimal invasive surgery, or in the field of non medical applications like electronic commerce, entertainment and education.

Acknowledgement. This work is funded by the German federal ministry of education and research (BMBF). Financial support is gratefully acknowledged. 


\section{References}

[1] Pesavento A., Lorenz A., Siebers S., Ermert H.: New real-time strain imaging concepts using diagnostic ultrasound. Phys. Med. Biol., Vol. 45, (2000), pp. 1423-1435.

[2] Khaled W., Ermert H., Bruhns O., Reichling S., Böse. H., Baumann M., Monkman G. J., Egersdörfer S., Meier A., Klein D., Freimuth H. - A haptic sensor-actor-system Studies in health technology and informatics, MMVR 11, Vol. 94 (2003), pp. 144-150

[3] Monkman G.J.: An Electrorheological Tactile Display - Presence. Journal of Teleoperators and Virtual Environments, MIT Press , Vol. 1, issue 2, (1992), pp. 219-228.

[4] Monkman G.J., Böse H., Ermert H., Khaled W., Klein D., Freimuth H., Baumann M., Egersdörfer S., Bruhns O.T., Meier A., Raja K.: Smart Fluid Based Haptic System for Telemedicine. 7th International conf. on the medical Aspects of telemedicine, Regensburg, Germany, (2002), pp. 59-70

[5] Pesavento A., Perrey C., Krueger M., Ermert H.: A time-efficient and accurate strain estimation concept for ultrasonic elastography using iterative phase zero estimation. IEEE Trans. Ultrasonics, Ferroelectrics and Frequency Control, vol. 46 (1999), 1057-1067.

[6] Scheipers U., Lorenz A., Pesavento A., Ermert H., Sommerfeld H.-J., Garcia-Schürmann M., Kühne K., Senge T., Philippou S.: Ultrasonic multifeature tissue characterization for the early detection of prostate cancer. IEEE Ultrasonics Symposium, (2001), pp. 1265-8

[7] Egevad L, Norberg M, Mattson S, Norlen B J and Busch C: Estimation of prostate cancer volume by multiple core biopsies before radical prostatectomy- Urology Vol. 52, (1998), pp. 653-8.

[8] Monkman G.J.: Addition of solid structures to electrorheological fluids. Journal of Rheology, Vol. 35, (Oct. 1991), pp. 1385-7.

[9] Böse. H, Monkman G. J., Freimuth H., Klein D., Ermert H., Baumann M., Egersdörfer S., Khaled W., Bruhns O. T. - ER Fluid Based Haptic System for Virtual Reality - 8th Interntional conf. on new Actuators, Bremen, (2002), pp. 351-354.

[10] Monkman G. J., Meier A. and Egersdörfer S. - Opto-isolated High voltage control arrayUK/European patent pending (March 2003) 